\title{
A Planar Wideband Microstrip Patch Antenna for UHF RFID Tag
}

\author{
M. S. R. Bashri ${ }^{1}$, M. I. Ibrahimy ${ }^{2}$, S. M. A. Motakabber ${ }^{3}$ \\ Department of Electrical and Computer Engineering, International Islamic University Malaysia (IIUM) \\ 53100 Kuala Lumpur, Malaysia \\ ${ }^{1}$ mohdsaifulriza@yahoo.com \\ ibrahimy@iium.edu.my \\ 3amotakabber@iium.edu.my
}

\begin{abstract}
In this research, a planar wideband microstrip patch antenna for passive radio frequency identification (RFID) tag is proposed. To enable universal operation by covering the operating frequency of ultra-high frequency (UHF) RFID band from $860 \mathrm{MHz}$ to $960 \mathrm{MHz}$, two C-shaped patches are employed. Both of the patches are inductively coupled fed by a rectangular loop feeding network for complex impedance matching with the referenced microchip impedance. The proposed antenna is designed for tagging metallic objects. To simplify the fabrication process and to reduce cost, a planar antenna structure is chosen over multi or cross layered configuration. The simulation and measurement results show impedance bandwidth of $159 \mathrm{MHz}$ and $155 \mathrm{MHz}$ (Return loss $\geq 3 \mathrm{~dB}$ ) respectively when mounted on metal plate.
\end{abstract}

Index Terms - Complex impedance matching, metallic objects, patch antenna, radio frequency identification (RFID), ultra-high frequency (UHF).

\section{INTRODUCTION}

The need for automatic monitoring and tracking of objects, animal or even human has led to intense research in radio frequency identification (RFID). With the ultimate aim to ease the process and to reduce as much as possible physical human intervention, RFID is seen as the way forward to realize the goal. Generally, an object is tagged with a wireless device called tag [1]. A reader located within the vicinity of the tagged objects read the information contained within the tag. The data carried by the tag consists of its identification number, location information and product specification [2]. Some of the advantages of RFID as compared to other automatic identification (Auto ID) technology such as optical barcode are independent of line of sight (LOS), high transmission rate and large storage capacity [3].

RFID system can be categorized into several groups based on its operating frequency, power source and protocol that govern its communication. There are four main operating frequency bands of RFID which are low frequency (LF), high frequency (HF), ultra high frequency (UHF) and microwave. LF and HF systems operate in the near field region by mean of inductive coupling between the tag and reader's coils hence resulting in shorter read range of about 1 meter [4]. On the other hand, UHF and microwave systems communicate via travelling electromagnetic coupling thus able to provide longer read range. In both systems, tag and reader are equipped with its own antenna. Since most of the emerging RFID applications require high read range, UHF system is rapidly becoming the preferred solution especially in supply chain management, logistics, and access control.

In UHF system, tag is made up of a microchip connected to an antenna as illustrated in Fig. 1. Tag is classified into three main categories which are active, semi-active and passive tag [3]. Active tag is equipped with its own power source such as battery to provide ample power to microchip and transmitter for tag to reader communication. However, the inclusion of battery and transmitter increases the cost of the tag substantially which might deter its implementation in low cost RFID solutions. In this case, passive tag can be used where it operates in the absence of both battery and transmitter. To provide power to microchip for this passive system, energy from the transmitted electromagnetic wave generated by reader is harvested by the tag antenna. As for tag to reader communication, a unique type modulation technique called backscattering modulation is used. This modulation is executed by the microchip by changing its front end complex RF input impedance between match and mismatch with the antenna input impedance corresponds to information bit ' 0 ' and ' 1 ' [1]. The resulting reflected power received by the reader varies between high and low which enable demodulation of the transmitted information from the tag. Semi-active tag lies between both active and passive where it has its internal power source albeit without transmitter. To compensate for the exclusion of transmitter, backscattering modulation method is also being used by semi-active tag.

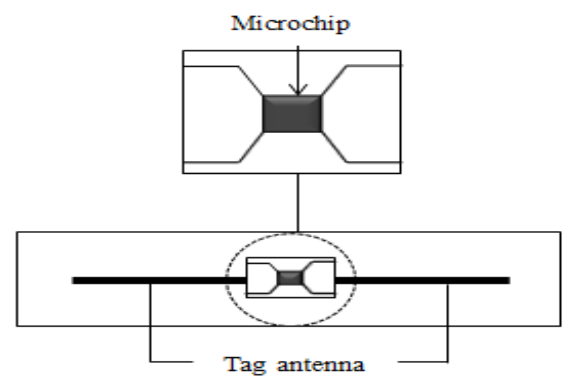

Fig.1 Typical RFID tag configuration 
A label-type printed dipole antenna is commonly adopted as a tag antenna due to its cheap cost and easy to fabricate [5-7]. However, there is limitation faced by this type of antenna which is serious performance degradation when mounted on metallic objects. This is due to current induced in the antenna is cancelled by the opposite image current induced in the metallic objects since at the surface of the perfect electric conductor (PEC), the tangential component of electric field cannot exist $[8,9]$. This scenario lead to changes in the antenna performances like shift in operating frequency thus leads to impedance mismatch, distorted radiation pattern and reduce gain.

To mitigate the issue, several methods have been proposed. One of them is the use of foam spacer to distance the tag and the metallic objects [10]. The tag is properly located at a distance where constructive interference occurs. However, the main limitation of the proposed solution is bulky tag configuration. In order to realize low profile structure, microstrip patch antenna has been given serious consideration due to the fact that it is naturally a grounded antenna. As a result, when it is used as tag antenna for metallic application, the surface of the objects will merely act as the antenna extended ground plane hence little performance changes is anticipated. Several patch antennas for metallic application has been presented with various patch shapes [11-13]. However, they are narrowband antenna thus not able to operate worldwide. To improve the bandwidth of the patch antenna, [14] and [15] has proposed microstrip antenna employing embedded slot design. However, these designs require cross layered structure due to via hole and shorting plate. Moreover, patch antenna incorporating dual layer substrate was proposed in [16]. All of these patch antenna designs presents complex and costly solution. In order to reduce fabrication complexity and cost, a simple and planar patch antenna configuration is needed. [17] has presented patch antenna for UHF RFID tag that does not require cross layered design. Although the fabrication process is greatly simplified, the antenna exhibit narrow band width thus limiting it use for universal tag application.

In this paper, a planar wideband microstrip patch antenna is proposed for UHF RFID tag for metallic application. Due to its wideband characteristic, it is aimed the proposed antenna is able to operate worldwide. Another interesting characteristic of the antenna is its planar structure that provides ease of fabrication and potential cost reduction. The rest of the paper is organised as follows. Section 2 discusses the antenna design. Section 3 presents the simulation and experimental results and discussion while conclusion is made in the last section.

\section{ANTENNA DESIGN}

Antenna design for UHF RFID system has been comprehensively presented in $[1,4]$. One of the important steps in tag antenna design is impedance matching. Unlike typical antenna where it is designed to match with the $50 \mathrm{ohm}$ feeding line such as coaxial cable, tag antenna for UHF systems is conjugate matched to complex impedance of the microchip connected to the antenna. To calculate the impedance matching, return loss (RL) of the antenna can be calculated using equation (1) [18]

$$
R L=-20 \log _{10}|\Gamma|
$$

where $\Gamma$ is the reflection coefficient at the antenna input terminal. $\Gamma$ can be expressed using equation (2) [19]

$$
\Gamma=\frac{Z_{\text {in }}-Z_{\text {chip }}^{*}}{Z_{\text {in }}+Z_{\text {chip }}}
$$

where $Z_{\text {in }}$ and $Z_{\text {chip }}$ are the complex input impedance of the tag antenna and the microchip.

In this design, Alien Higgs-3 is chosen as the referenced microchip. The complex impedance of the microchip is, $Z_{\text {chip }}=31-212 \Omega$ at $915 \mathrm{MHz}$. The capacitive reactance of the microchip is due to the inclusion of energy-storage stage in the microchip design. In order to present an inductive reactance at the antenna input impedance so as to cancel the capacitive reactance of the microchip, a rectangular loop patch is chosen as the feeding network. The feed loop acts a transformer to transform the impedance of a radiating element which is in close proximity with the loop. The input terminal of the rectangular loop is directly connected to the microchip. The input impedance seen at the input terminal of the feeding loop is given by [20]

$$
Z_{\text {in }}=Z_{\text {loop }}+\frac{(2 \pi f M)^{2}}{Z_{r b}}
$$

where $Z_{\text {loop }}, M$ and $Z_{r b}$ are the input impedance of the loop, mutual inductance between the loop and the radiating element and input impedance of the radiating element. Based on equation (3), the input resistance, $R_{\text {in }}$ and input reactance, $X_{\text {in }}$ are individually expressed in equation (4) and equation (5)

$$
\begin{aligned}
& R_{\text {in }}\left(f_{0}\right)=\frac{\left(2 \pi f_{0} M\right)^{2}}{R_{r b}\left(f_{0}\right)} \\
& X_{\text {in }}\left(f_{0}\right)=2 \pi f_{0} L_{\text {loop }}
\end{aligned}
$$

where $f_{0}$ is the operating frequency of the antenna. Based on equation (4), the input resistance depends on mutual inductance between the loop and the radiating element and can be controlled by varying the loop size and the distance between the loop and the radiating elements. As for the input reactance, it is mainly affected by the loop's parameter as shown in equation (6) [21]

$$
L_{\text {loop }}=0.4\left(L_{L}+W_{L}\right) \ln \left[\frac{2 L_{L} W_{L}}{s\left(L_{L}+W_{L}\right)}\right](\mu H)
$$


Initially, rectangular patch antenna is used as the radiating element for the design due to its simple structure and easy to analyse. The length of the rectangular patch is firstly approximated using closed form expression below [22]

$$
L_{e f f}=L+\Delta L
$$

where $L_{e f f}, L$ and $\Delta L$ are the effective length, physical length and the extended length due to fringing field effects. $\Delta L$ is given by [22]

$$
\frac{\Delta L}{h}=0.412 \frac{\left(\varepsilon_{e f f}+0.3\right)\left(\frac{W}{h}+0.264\right)}{\left(\varepsilon_{e f f}-0.258\right)\left(\frac{W}{h}+0.8\right)}
$$

$W$ is the width of the rectangular patch, $h$ is the substrate height and $\varepsilon_{\text {eff }}$ is the effective dielectric constant. For the case $W / h>1$, $\varepsilon_{e f f}$ can be calculated using equation (11) as follows [23]

$$
\varepsilon_{e f f}=\frac{\varepsilon_{r}+1}{2}+\frac{\varepsilon_{r}-1}{2}\left[1+12 \frac{h}{W}\right]^{-1 / 2}
$$

$\varepsilon_{r}$ is the relative dielectric constant of the substrate.

The substrate for the patch antenna is glass epoxy FR-4 with dielectric constant, $\varepsilon_{r}=4.4$ and tangential loss, $\tan \delta=$ 0.002 . The selection of the substrate is made due to its cheap cost to reduce the antenna cost. The thickness of the substrate, $h$ was set to $1.6 \mathrm{~mm}$ to keep the antenna low profile. To realize wideband characteristic, multi resonator configuration is implemented where two patches are fed by the same loop feeding network as shown in Fig. 2.

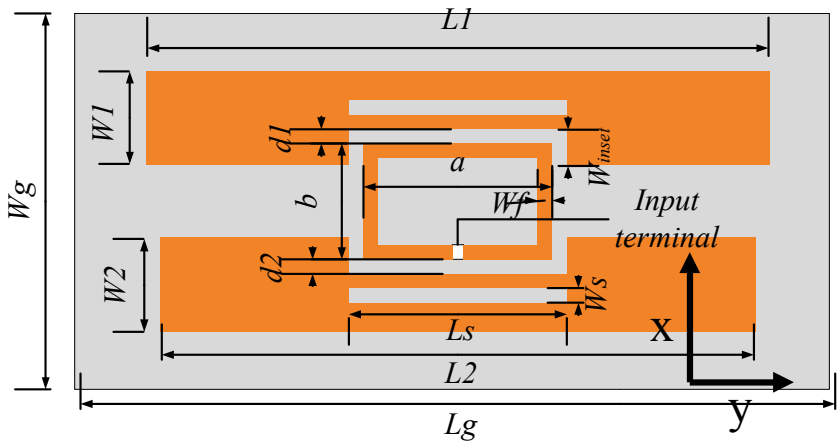

(a)

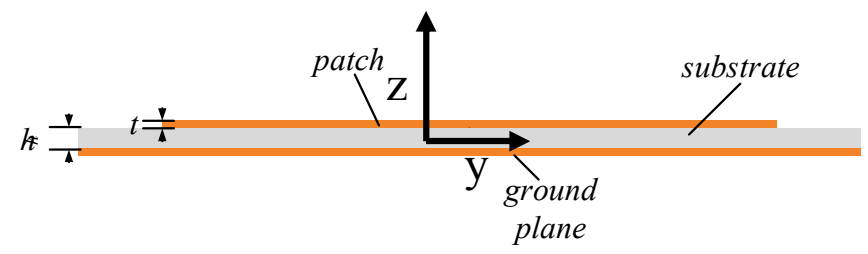

(b)

Fig.2 The geometry of the antenna. (a) Top view and (b) side view.
The width of both radiating element is fixed to $10 \mathrm{~mm}$ to ensure small size antenna is achieved. A rectangular slot is then cut at one of the non-radiating side of the rectangular patches to further reduce the antenna dimension. This leads to increase of electrical current path on the patches thus lowering the resonant frequency hence making the projected length shorter than that of initial rectangular form [23]. In addition, two narrow slots are embedded at both of the resulting C-shaped patches for easy tuning of the resonating frequency without the need to change other parameters.

The antenna is constructed using the approximated calculated parameter value using equation (4)-(11). Afterwards, parametric refinement is carried out to produce the optimal antenna design. The optimal antenna parameter is tabulated in Table 1. The prototype of the antenna is illustrated in Fig. 3.

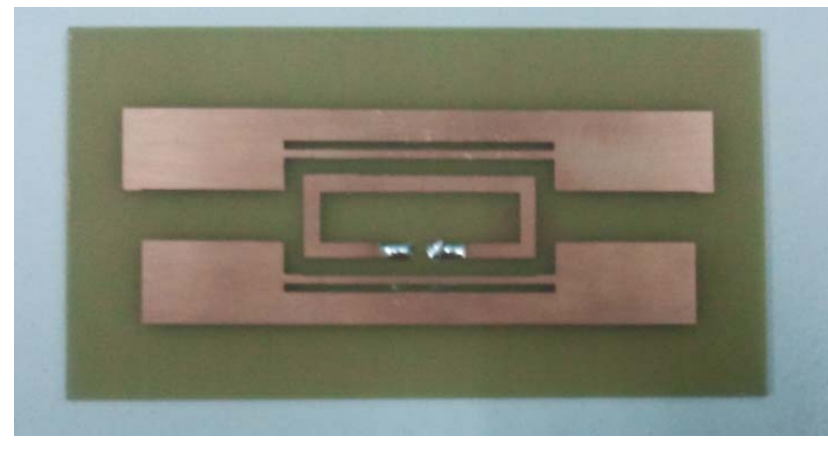

Fig. 3 Prototype of the proposed wideband antenna.

TABLE 1

\begin{tabular}{|c|c|}
\hline Parameter & Value (mm) \\
\hline$W 1$ & 10 \\
\hline$L 1$ & 74 \\
\hline$W 2$ & 10 \\
\hline$L 2$ & 69 \\
\hline$W_{s}$ & 1 \\
\hline$L_{s}$ & 30 \\
\hline$d 1$ & 2 \\
\hline$d 2$ & 2 \\
\hline$W_{\text {inset }}$ & 4 \\
\hline$t$ & 0.0358 \\
\hline$h$ & 1.6 \\
\hline$W_{f}$ & 2 \\
\hline$a$ & 29 \\
\hline$b$ & 10 \\
\hline Ground plane and substrate & $87 \times 45$ \\
\hline
\end{tabular}

OPTIMIZED DESIGN SPECIFICATION OF THE PROPOSED ANTENNA 


\section{RESULTS AND DISCUSSION}

The surface current density of the antenna at both resonant frequencies is illustrated in Fig. 4. It is clearly shown that the antenna resonates at two different frequencies close to each other to form wide impedance bandwidth. To observe the effect of metallic surface on the proposed antenna performance, simulation and measurement of the antenna when placed on the metal plate was also performed.

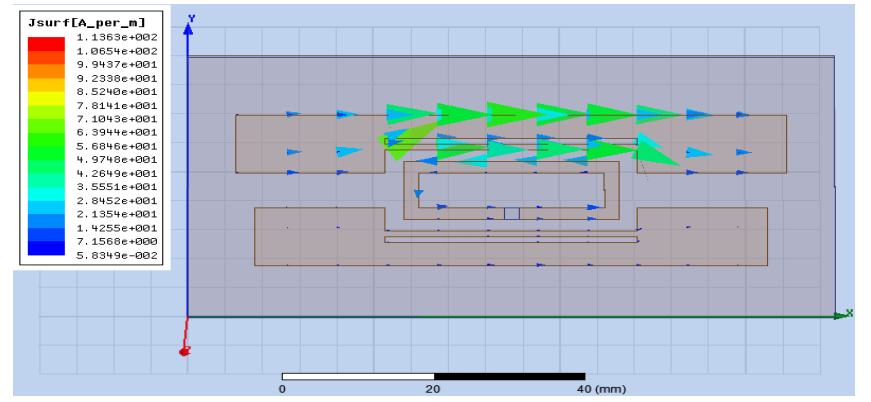

(a)

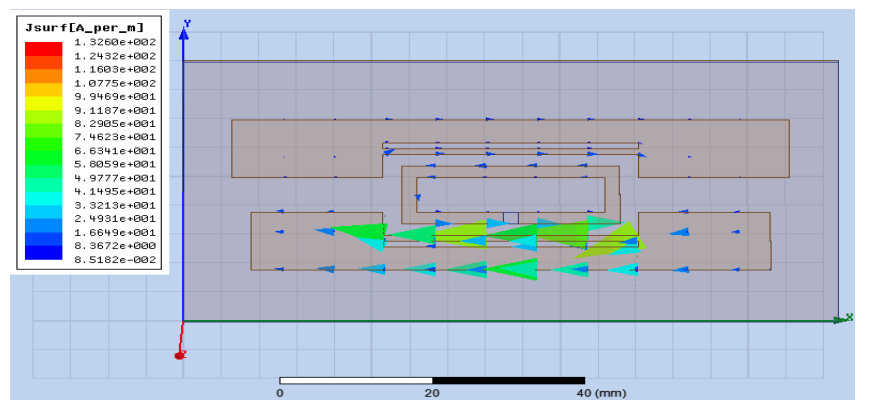

(b)

Fig. 4 Surface current distribution of the antenna at two resonant modes. (a) $883 \mathrm{MHz}$ and (b) $953 \mathrm{MHz}$.

The simulated and measured impedance of the antenna for the two scenarios are depicted in Fig. 5. A good agreement between the two results proves that good impedance matching is realized throughout the entire UHF frequency band. The different between the simulation and measurement results especially for the antenna input resistance is due to fabrication inaccuracies and the surrounding effects during the measurement process. Nevertheless, the half-power impedance bandwidth ( $R L \geq 3 \mathrm{~dB}$ ) for both cases is well above the required $100 \mathrm{MHz}$ as shown in Fig. 6.

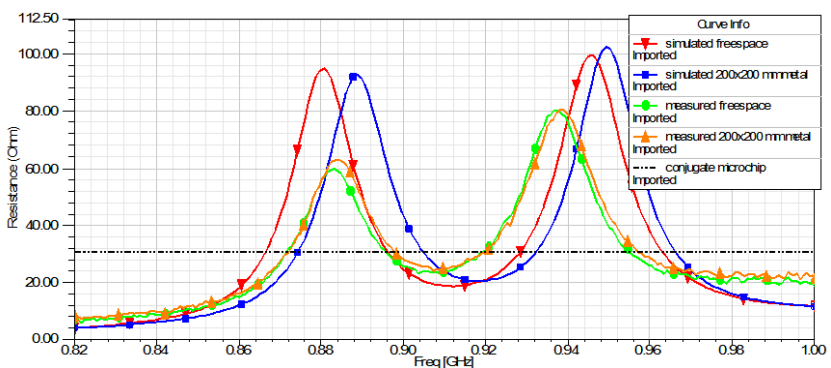

(a)

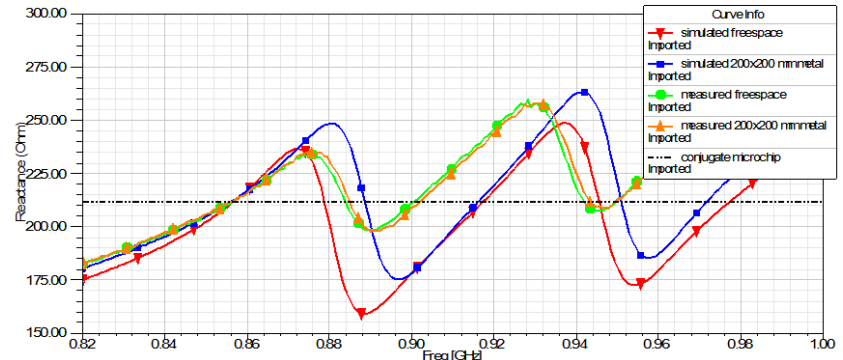

(b)

Fig. 5 Simulated and measured impedance of the antenna and the microchip conjugate impedance value against frequency. (a) Resistance and (b) reactance value against frequency.

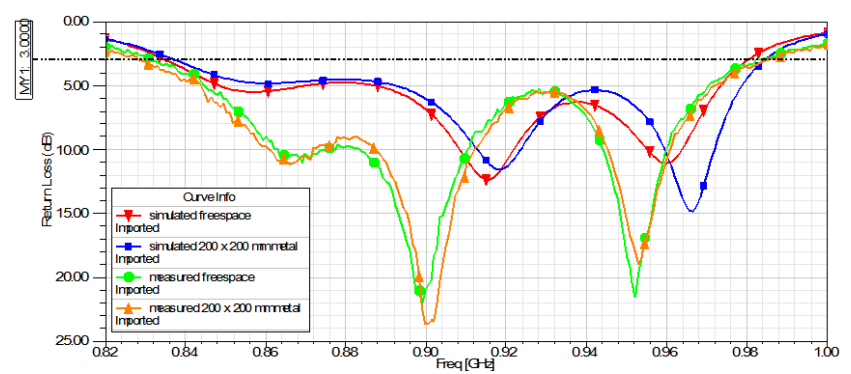

Fig. 6 Return loss $(\mathrm{dB})$ of the proposed antenna.

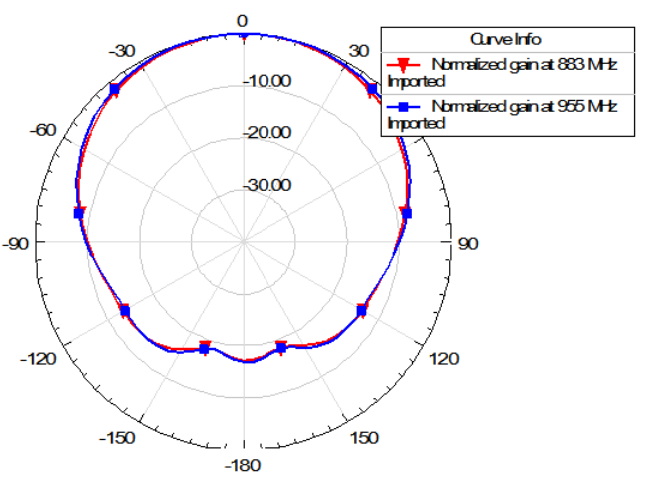

(a)

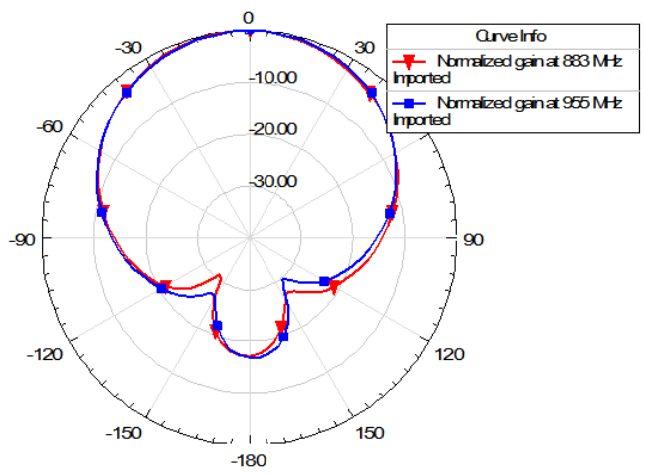

(b)

Fig. 7 Simulated (a) normalized E-field and (b) normalized H-field radiation patterns at two resonant frequencies. 
The simulated radiation patterns of the antenna at the resonating frequencies are shown in Fig. 7. The resulting patterns at both of the resonance are almost identical and exhibit broadside radiation pattern as expected of microstrip patch antenna. Furthermore, the peak gain of the antenna is illustrated in Fig. 8.

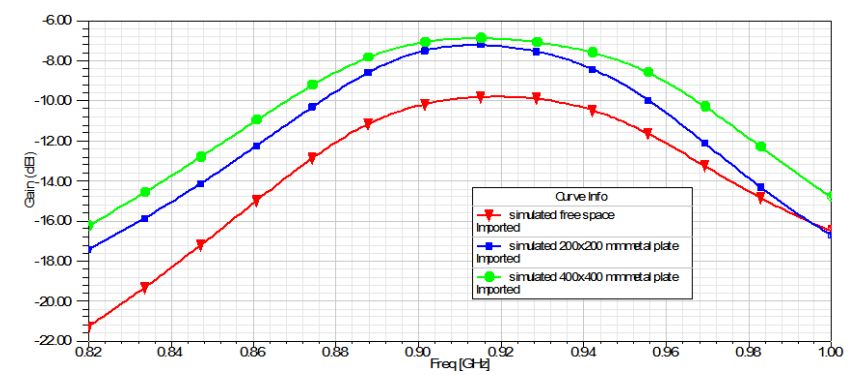

Fig. 8 Peak antenna gain of the proposed antenna.

\section{CONCLUSIONS}

A planar wideband microstrip patch antenna is proposed in this research. Two C-shaped patches are fed by rectangular loop feeding structure to increase the bandwidth of the antenna for universal usage. The planar structure of the antenna provides ease of fabrication due to the exclusion of multi and cross layered configuration. The design of the antenna is supplemented by simulation and experimental results. The simulated and measured half power bandwidths of the antenna $(\mathrm{RL} \geq 3 \mathrm{~dB})$ are $159 \mathrm{MHz}$ and $155 \mathrm{MHz}$ respectively. Moreover, the peak gain of the antenna is sufficient to give reasonable read range for the entire UHF frequency band. In the future, measurement for the antenna radiation pattern shall be conducted to complement the simulation results.

\section{REFERENCES}

K. V. S. Rao, P. V. Nikitin, and S. F. Lam, "Antenna Design for UHF RFID Tags: A Review and a Practical Application," IEEE Transactions on Antennas and Propagation, vol. 53, pp. 3870-3876, 2005.

[2] K. Finkenzeller, RFID Handbook, 2nd ed. West Sussex: John Wiley \& Sons, 2003.

[3] D. M. Dobkin, The RF in RFID : Passive UHF RFID in Practice. Massachusetts: Elsevier Inc., 2008

[4] G. Marrocco, "The art of UHF RFID antenna design: impedancematching and size-reduction techniques " IEEE Antennas and Propagation Magazine, vol. 50, pp. 66-79, 2008.

[5] W. Choi, H. W. Son, C. Shin, J.-H. Bae, and G. Choi, "RFID tag antenna with a meandered dipole and inductively coupled feed," presented at the IEEE Antennas and Propagation Society International Symposium, 2006.

[6] Z. Fang, R. Jin, and J. Geng, "Asymmetric dipole antenna suitable for active RFID tags," Electronics Letters, vol. 44, pp. 71-72, 2008. G. Monti, L. Catarinucci, and L. Tarricone, "Broad-band dipole for RFID applications," Progress In Electromagnetics Research C, vol. 12, pp. 163-172, 2010.

[8] N. Ghannay, M. B. Ben Salah, F. Romdhani, and A. Samet, "Effects of metal plate to RFID tag antenna parameters," presented at the Microwave Symposium Mediterrannean (MMS), 2009.

[9] J. T. Prothro, G. D. Durgin, and J. D. Griffin, "The Effects of a Metal Ground Plane on RFID Tag Antennas," presented at the IEEE Antennas and Propagation Society International Symposium 2006.
[10] C. Cho, H. Choo, and I. Park, "Design of planar RFID tag antenna for metallic objects," Electronics Letters, vol. 44, pp. 175-177, 2008

[11] J. Yan, Q. Feng, C. Li, and Q. Li;, "A novel patch antenna for UHF band RFID tag " presented at the 7th International Conference On Wireless And Optical Communications Networks (WOCN), 2010.

[12] H.-W. Son, G.-Y. Choi, and C.-S. Pyo, "Design of wideband RFID tag antenna for metallic surfaces " Electronics Letters, vol. 42, pp. 263-265, 2006.

[13] H.-W. Son and S.-H. Jeong, "Wideband RFID Tag Antenna for Metallic Surfaces Using Proximity-Coupled Feed " IEEE Antennas and Wireless Propagation Letters, vol. 10, pp. 377-380, 2011.

[14] J. Z. Huang, P. H. Yang, W. C. Chew, and T. T. Ye, "A compact broadband patch antenna for UHF RFID tags " presented at the Asia Pacific Microwave Conference (APMC), 2009.

[15] J.-H. Lu and K.-T. Hung, "Planar UHF RFID tag antenna with bandwidth enhancement " presented at the Cross Strait QuadRegional Radio Science and Wireless Technology Conference (CSQRWC), Harbin, 2011.

[16] W. Choi, H. W. Son, J.-H. Bae, G. Y. Choi, C. S. Pyo, and J.-S. Chae, "An RFID Tag Using a Planar Inverted-F Antenna Capable of Being Stuck to Metallic Objects," ETRI Journal, vol. 20, pp. 216218, 2006 .

[17] T. Tashi, M. S. Hasan, and H. Yu, "A complete planner design of microstrip patch antenna for a passive UHF RFID tag," presented at the 17th International Conference on Automation and Computing (ICAC), 2011.

[18] T. S. Bird, "Definition and Misuse of Return Loss," IEEE Antennas and Propagation Magazine, vol. 51, pp. 166-167, 2009.

[19] C.-H. Loo, K. Elmahgoub, F. Yang, A. Z. Elsherbeni, D. Kajfez, A. A. Kishk, T. Elsherbeni, L. Ukkonen, L. Sydanheimo, M. Kivikoski, S. Merilampi, and P. Ruuskanen, "Chip impedance matching for UHF RFID tag antenna design," Progress In Electromagnetics Research, vol. 81, pp. 359-370, 2008.

[20] H.-W. Son and C.-S. Pyo, "Design of RFID tag antennas using an inductively coupled feed," Electronics Letters, vol. 41, pp. 994-996, 2005.

[21] P. H. Yang, Y. Li, L. Jiang, W. C. Chew, and T. T. Ye, "Compact Metallic RFID Tag Antennas With a Loop-Fed Method " IEEE Transactions on Antennas and Propagation, vol. 59, pp. 4454-4462, 2011.

[22] C. A. Balanis, Antenna Theory Analysis and Design, 3rd ed. New Jersey: John Wiley \& Sons, 2005.

[23] G. Kumar and K. P. Ray, Broadband Microstrip Antenna. Noorwood, MA: Artech House, 2003. 